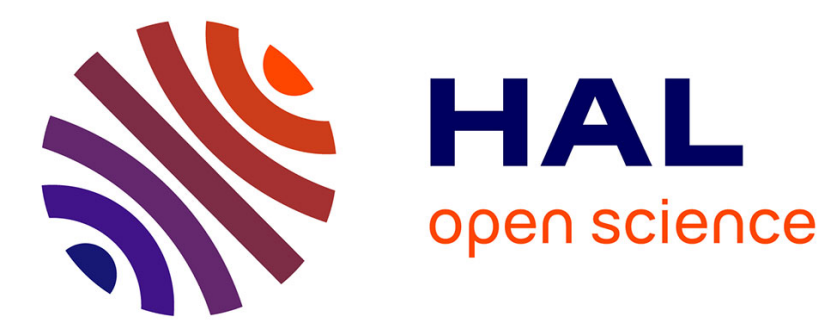

\title{
Acyclic Coloring of Graphs of Maximum Degree Five: Nine Colors are Enough
}

\author{
Guillaume Fertin, André Raspaud
}

\section{To cite this version:}

Guillaume Fertin, André Raspaud. Acyclic Coloring of Graphs of Maximum Degree Five: Nine Colors are Enough. Information Processing Letters, 2008, 105 (2), pp.65-72. hal-00416567

\section{HAL Id: hal-00416567 \\ https://hal.science/hal-00416567}

Submitted on 15 Sep 2009

HAL is a multi-disciplinary open access archive for the deposit and dissemination of scientific research documents, whether they are published or not. The documents may come from teaching and research institutions in France or abroad, or from public or private research centers.
L'archive ouverte pluridisciplinaire HAL, est destinée au dépôt et à la diffusion de documents scientifiques de niveau recherche, publiés ou non, émanant des établissements d'enseignement et de recherche français ou étrangers, des laboratoires publics ou privés. 


\title{
Acyclic Coloring of Graphs of Maximum Degree Five: Nine Colors are Enough
}

\author{
Guillaume Fertin ${ }^{1}$, André Raspaud ${ }^{2}$ \\ ${ }^{1}$ LINA, FRE CNRS 2729, Université de Nantes \\ 2 rue de la Houssinière - BP 92208 - F44322 Nantes Cedex 3 \\ ${ }^{2}$ LaBRI UMR CNRS 5800, Université Bordeaux 1 \\ 351 Cours de la Libération - F33405 Talence Cedex \\ fertin@lina.univ-nantes.fr, raspaud@labri.fr
}

\begin{abstract}
An acyclic coloring of a graph $G$ is a coloring of its vertices such that: (i) no two neighbors in $G$ are assigned the same color and (ii) no bicolored cycle can exist in $G$. The acyclic chromatic number of $G$ is the least number of colors necessary to acyclically color $G$. In this paper, we show that any graph of maximum degree 5 has acyclic chromatic number at most 9 , and we give a linear time algorithm that achieves this bound.
\end{abstract}

Keywords: acyclic chromatic number, acyclic coloring algorithm, maximum degree 5 .

\section{Introduction}

In this paper, we address the acyclic coloring problem. An acyclic coloring of a graph $G$ is a coloring of its vertices such that: (i) no two neighbors in $G$ are assigned the same color (this condition ensures a proper coloring of $G$ ) and (ii) no bicolored cycle can exist in $G$. In other words, an acyclic coloring of $G$ is a proper coloring of $G$ such that any two classes of colors induce a graph $G^{\prime}$ which is a forest (that is, an acyclic graph). The minimum number of colors necessary to acyclically color $G$ is called the acyclic chromatic number of $G$, and is noted $a(G)$.

For a family $\mathcal{F}$ of graphs, the acyclic chromatic number of $\mathcal{F}$, denoted by $a(\mathcal{F})$, is defined as the maximum $a(G)$ over all graphs $G \in \mathcal{F}$. Acyclic coloring has been largely studied in the recent past ; in particular, $a(\mathcal{F})$ has been determined for several families $\mathcal{F}$ of graphs such as planar graphs [Bor79], planar graphs with "large" girth [BKW99], 1-planar graphs [BKRS01], outerplanar graphs (see for instance [Sop97]), product of trees [JMV06] and $d$-dimensional grids [FGR03]. Concerning graphs having a fixed maximum degree, most of the results on this topic come from Alon et al. [AMR90], where the following results were proved: (1) asymptotically, there exist graphs of maximum degree $\Delta$ with acyclic chromatic number in $\Omega\left(\frac{\Delta^{\frac{4}{3}}}{(\log \Delta)^{\frac{1}{3}}}\right) ;(2)$ asymptotically, it is possible to acyclically color any graph of maximum degree $\Delta$ with $O\left(\Delta^{\frac{4}{3}}\right)$ colors ; (3) a trivial greedy polynomial time algorithm exists that acyclically colors any graph of maximum degree $\Delta$ in $\Delta^{2}+1$ colors. We note that the main drawback concerning results (1) and (2) is that they come from probabilistic arguments, whose proofs are existential but not constructive.

Now, for fixed values of $\Delta$, it has been shown that 4 colors are sufficient to color any graph of maximum degree 3 [Grü73], and that there exists a graph $G$ of maximum degree 3 for which any acyclic coloring requires 4 colors (a trivial example is the complete graph $K_{4}$ ). Moreover, it was proved recently in [Sku04] that there exists a linear time algorithm that acyclically colors any graph of maximum degree 3 in 4 colors. It has also been shown in [Bur79] that 5 colors are 
sufficient to color any graph of maximum degree 4 , and there exists a graph $G$ of maximum degree 4 for which any acyclic coloring requires 5 colors (a trivial example is the complete graph $K_{5}$ ).

The main result presented in this paper can be seen as a follow-up of the cases $\Delta=3$ and $\Delta=4$. Indeed, for $\Delta=5$, we show that any graph of degree 5 can be acyclically colored in 9 colors ; besides, we design a linear-time algorithm that achieves such a bound.

\section{Preliminaries}

In the following, we will only consider graphs $G$ of maximum degree $\Delta=5$. A partial coloring of $G$ will denote a coloring of a set $V_{c} \subseteq V$ of vertices of $G$, such that the graph $G\left[V_{c}\right]$ induced by $V_{c}$ is acyclically colored. We will then say that $G$ is partially colored. If $G$ is partially colored by a set of colors of cardinality $k$, we will say that $G$ uses $k$ colors.

Consider a partially colored graph $G$, and consider an uncolored vertex $u \in V-V_{c}$. Let $N(u)$ be the set of the neighbors of $u$. In the following, we will be interested of the set $N_{c}(u) \subseteq$ $N(u)$ of colored vertices in $N(u)$. We will denote by \#cn $(u)$ (number of colored neighbors of $u$ ) the cardinality of $N_{c}(u)$. We will also be interested in the set of colors $S C\left(N_{c}(u)\right)$ used by the vertices in $N_{c}(u)$, and we will denote by \#dcn(u) (different colors in the neighborhood of $u$ ) the cardinality of $S C\left(N_{c}(u)\right)$. We will also find convenient to use the following notation: $L_{u}=\left(n_{1}, n_{2}, \ldots n_{\# d c n(u)}\right)$ (where $n_{1} \geq n_{2} \geq \ldots \geq n_{\# d c n(u)}$ ), where an $n_{i}$ represents, for a color $c$ in $S C\left(N_{c}(u)\right)$, the number of times $c$ is used among the colored neighbors of $u$. We will then say than vertex $u$ sees list $L_{u}=\left(n_{1}, n_{2}, \ldots n_{\# d c n(u)}\right)$, and $L_{u}$ will be called the color list of $u$. An illustration of those notions is given in Figure 1: here, all the neighbors of $u$ are colored (thus $\# c n(u)=5$ and $\left.N_{c}(u)=N(u)\right), S C\left(N_{c}(u)\right)=\left\{c_{1}, c_{2}, c_{3}\right\}$, \#dcn $(u)=3$ and $L_{u}=(2,2,1)$.

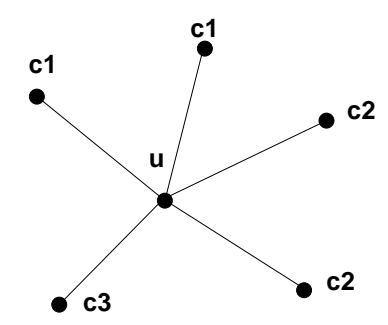

Figure 1: Vertex $u$ sees $L_{u}=(2,2,1)$

We will also focus on the possibility that coloring $u$ with a given color $c^{\prime}$ induces a bicolored cycle in graph $G$. Hence, for two distinct colors $c$ and $c^{\prime}$, a bicolored cycle of the form $\left(c, c^{\prime}\right)$ will denote a bicolored cycle whose colors are $c$ and $c^{\prime}$. Moreover, for two distinct colors $c$ and $c^{\prime}$ and for any uncolored vertex $u$, we introduce a boolean variable $N\left(c, c^{\prime}, u\right)$ defined as follows: $N\left(c, c^{\prime}, u\right)=0$ if we can ensure that assigning color $c^{\prime}$ to $u$ does not create a bicolored cycle of the form $\left(c, c^{\prime}\right)$, and $N\left(c, c^{\prime}, u\right)=1$ otherwise. More intuitively, if two distinct neighbors $v_{1}$ and $v_{2}$ of $u$ are assigned color $c$, and if both $v_{1}$ and $v_{2}$ have a colored neighbor with color $c^{\prime}$, then coloring $u$ with color $c^{\prime}$ might create a bicolored cycle of the form $\left(c, c^{\prime}\right)$ (because we have a bicolored path of length 4 going through $u$ ), and thus we will set $N\left(c, c^{\prime}, u\right)$ to 1 .

Finally, we will often find convenient to denote, for a colored vertex $v$ of $G$, by $c(v)$ the color that is assigned to $v$.

\section{Acyclic Coloring of Graphs of Maximum Degree 5}

In this section, we show that any graph of maximum degree 5 has acyclic chromatic number at most 9, and we give a linear time algorithm to achieve this bound (see Theorem 1). This result will be obtained thanks to Lemmas 1 to 5 . Before proving those lemmas, we begin by a first observation. 
Observation 1 Let $G$ be a partially colored graph of maximum degree 5. Let $u$ be an uncolored vertex in $G$, and let us consider a given color $c$ in $S C\left(N_{c}(u)\right)$. Let $n_{c}$ be the number of neighbors of $u$ having color $c$. In that case, if $n_{c}=1$, then for any color $c^{\prime} \neq c, N\left(c, c^{\prime}, u\right)=0$. Moreover, if $n_{c} \geq 2$, then there exists at most $2 n_{c}$ distinct colors $c^{\prime} \neq c$ for which $N\left(c, c^{\prime}, u\right)=1$.

Proof: Clearly, since $G$ is partially colored, there is no bicolored cycle in $G$, and assigning a color to $u$ could create one or several bicolored cycles. However, if for a color $c$ represented among the neighbors of $u$, we have $n_{c}=1$, then no bicolored cycle could involve $u$ and color $c$ (because no bicolored path carrying color $c$ could go through $u$ ), and thus for any $c^{\prime} \neq c$, we have $N\left(c, c^{\prime}, u\right)=0$.

Now, if $n_{c} \geq 2$, consider the $n_{c}$ neighbors of $u$ having color $c$, say $v_{1}, v_{2} \ldots v_{n_{c}}$. Each of those vertices has at most $\Delta-1=4$ colored neighbors. Coloring vertex $u$ with color $c^{\prime}$ could then contribute to a bicolored cycle if two distinct vertices $v_{i}$ and $v_{j}, 1 \leq i, j \leq n_{c}$ both have a neighbor having the same color $c^{\prime}$. More precisely, suppose $v_{i}$ (resp. $v_{j}$ ) has a neighbor $w_{i}$ (resp. $w_{j}$ ) having color $c^{\prime}$ (note that possibly, $w_{i}=w_{j}$ ). If we assign color $c^{\prime}$ to $u$, then we have a bicolored path of length $4,\left(w_{i}, v_{i}, u, v_{j}, w_{j}\right)$, and hence $u$ could contribute to a bicolored cycle of the form $\left(c, c^{\prime}\right)$. Now we need to compute an upper bound on the number of colors $c^{\prime}$ for which $N\left(c, c^{\prime}, u\right)=1$. We first note that since every neighbor of $u$ has at most 4 colored neighbors, then there can exist at most $4 n_{c}$ colored vertices which are neighbors of one of the $n_{c}$ neighbors of $u$ that carry color $c$. Moreover, any bicolored cycle of the form $\left(c, c^{\prime}\right)$ going through $u$ can exist only if at least 2 vertices at distance 2 from $u$ are assigned the same color $c^{\prime}$. Hence, we conclude that there are at most $2 n_{c}$ colors $c^{\prime}$ for which $N\left(c, c^{\prime}, u\right)=1$.

Observation 1 above implies the following: let $G$ be a partially colored graph, and let $u$ be an uncolored vertex. If one wishes to color $u$ in such a way that $G$ remains partially colored, then $u$ needs to avoid the following colors:

- the colors of its neighbors (in order to maintain a proper coloring for $G$ ).

- the colors $c^{\prime}$ mentioned in proof of Observation 1, knowing that there are at most $2 n_{c}$ such colors for every color $c$ that appears at least twice among the neighbors of $u$.

The above discussion will help us prove Lemma 1 below.

Lemma 1 Let $G$ be a partially colored graph of maximum degree 5, and let us suppose $G$ uses at most 9 colors. Then, for any uncolored vertex $u$ such that $\# c n(u) \leq 3$, there exists a way to color $u$ such that $G$ is still partially colored, and still uses at most 9 colors.

Proof: Suppose \#cn $(u) \leq 3$. If no color is repeated among the neighbors of $u$ (thus $L_{u}=(1)$, $L_{u}=(1,1)$ or $\left.L_{u}=(1,1,1)\right)$, then no bicolored cycle can be created by coloring $u$. Thus $u$ only needs to avoid the colors used by its neighbors, and since at most three colors are used, there exists at least 6 choices for coloring $u$. Now, suppose that at least one color is repeated among the neighbors of $u$. Then, since \#cn $(u) \leq 3$, we know that exactly one color is repeated among its neighbors. We have three cases: $L_{u}=(2), L_{u}=(2,1)$ or $L_{u}=(3)$. Let us detail those cases: Case 1: $L_{u}=(2,1)$ or $L_{u}=(2)$. Then, by Observation $1, u$ needs to avoid (i) 4 colors (no bicolored cycle) and (ii) at most 2 more colors (in order to maintain the proper coloring). Since we have 9 colors available, at least 3 choices remain for coloring $u$.

Case 2: $L_{u}=(3)$. Similarly to Case 1, $u$ needs to avoid (i) 6 colors (no bicolored cycles) and (ii) 1 more color (proper coloring). Hence, there remains 2 choices for coloring $u$.

Lemma 1 above handles the case where at most three neighbors of $u$ are colored. However, Observation 1 helps to solve more cases. This is the purpose of Lemma 2 below.

Lemma 2 Let $G$ be a partially colored graph of maximum degree 5, and let us suppose $G$ uses at most 9 colors. For any uncolored vertex $u$, if $L_{u}=(1,1,1,1), L_{u}=(1,1,1,1,1), L_{u}=(2,1,1)$, $L_{u}=(2,1,1,1)$ or $L_{u}=(3,1)$, then there exists a way to color $u$ such that $G$ is still partially colored, and still uses at most 9 colors. 
Proof: Let us detail the different cases:

Case 1: $L_{u}=(1,1,1,1,1)$ or $L_{u}=(1,1,1,1)$. Since no color is repeated among the neighbors of $u$, we only need maintain the proper coloring when coloring $u$; hence, $u$ needs to avoid at most 5 colors. Thus, there remains at least 4 choices for coloring $u$.

Case 2: $L_{u}=(2,1,1,1)$ or $L_{u}=(2,1,1)$. Then $u$ needs to avoid (i) 4 colors (no bicolored cycle, see Observation 1) and (ii) at most 4 more colors (proper coloring). Since we have 9 colors available, there remains at least one choice for coloring $u$.

Case 3: $L_{u}=(3,1)$. Then $u$ needs to avoid (i) 6 colors (no bicolored cycle, see Observation 1) and (ii) 2 more colors (proper coloring). Since we have 9 colors available, there remains one choice for coloring $u$.

Lemmas 1 and 2 can handle a certain number of cases, but not all of them. For instance, if $L_{u}=(3,1,1)$, the counting argument derived from Observation 1 yields that $u$ needs to avoid (i) 6 colors (no bicolored cycles, by Observation 1) and (ii) 3 more colors (proper coloring). Since we have only 9 colors available, we cannot ensure that it is possible to find a suitable color for $u$. Thus, we need a more detailed argument. The idea that we will exploit is the following: if there is a large number of colors that $u$ should avoid because of possible bicolored cycles, this is because there are colored neighbors of $u$ that have themselves many neighbors colored with distinct colors. That is, if a colored neighbor of $u$ has colored neighbors which are assigned the same color, then this will decrease the number of possible colors that $u$ should avoid. This idea is summarized in the following observation.

Observation 2 Let $G$ be a partially colored graph of maximum degree 5. Let $u$ be an uncolored vertex in $G$, and let us consider a given color $c$ in $S C\left(N_{c}(u)\right)$ such that $n_{c}$, the number of neighbors of $u$ having color $c$, is greater than or equal to 2. Let $v_{1}, \ldots v_{n_{c}}, 2 \leq n_{c} \leq 5$ be the neighbors of $u$ whose color is $c$. If, for every $1 \leq i \leq n_{c}$, there is at least one color which is repeated among the neighbors of $v_{i}$, then there exists at most $\left\lfloor\frac{3 n_{c}}{2}\right\rfloor$ distinct colors $c^{\prime} \neq c$ for which $N\left(c, c^{\prime}, u\right)=1$.

Proof: Let $c$ be a color which appears at least twice among the neighbors of $u$, and let $v_{1}, \ldots v_{n_{c}}$, $2 \leq n_{c} \leq 5$ be the neighbors of $u$ colored $c$. Suppose that for every $1 \leq i \leq n_{c}$, there is at least one color which is repeated among the neighbors of $v_{i}$. This means that at most 3 distinct colors are represented among the neighbors of $v_{i}$, for every $1 \leq i \leq n_{c}$. Take any of those colors, say $c^{\prime}$. If $c^{\prime}$ does not appear as neighbor of two distinct $v_{i}$ s, then $N\left(c, c^{\prime}, u\right)=0$. Thus, the number of colors $c^{\prime}$ for which $N\left(c, c^{\prime}, u\right)=1$ is upper bounded by $\frac{3 n_{c}}{2}$. Since this number is an integer, we conclude that it cannot exceed $\left\lfloor\frac{3 n_{c}}{2}\right\rfloor$.

Thanks to Observation 2, we can now solve more cases, as shown in Lemma 3.

Lemma 3 Let $G$ be a partially colored graph of maximum degree 5, and let us suppose $G$ uses at most 9 colors. For any uncolored vertex $u$, if $L_{u}=(2,2), L_{u}=(3,1,1), L_{u}=(4), L_{u}=(4,1)$ or $L_{u}=(5)$, then there exists a way to color $u$ such that $G$ is still partially colored, and still uses at most 9 colors.

Proof: Let us detail the possible cases:

Case 1: $L_{u}=(2,2)$. Let $c_{1}$ and $c_{2}$ be the two colors represented among the 4 colored neighbors of $u$, and let us distinguish two cases.

(1) If there exists a colored neighbor $v$ of $u$ such that the colored neighbors of $v$ use pairwise distinct colors, then it is possible to recolor $v$ : indeed, $v$ cannot be involved in a bicolored cycle, and since it has at most 4 colored neighbors (because $u$ is not colored yet), $v$ can be assigned five different colors, hence there are four other possibilities for coloring $v$. Among those four colors, we pick a color $c$ such that $c \neq c_{1}$ and $c \neq c_{2}$. In that case, $L_{u}$ is modified and becomes $L_{u}=(2,1,1)$, a case that is handled by Lemma 2 . 
(2) If any colored neighbor $v$ of $u$ is such that at least one color is repeated among the colored neighbors of $v$, then by Observation 2, we conclude that $u$ should avoid (i) 6 colors (no bicolored cycles) and (ii) 2 more colors (proper coloring). Since we have 9 colors, there is always a color available for $u$.

Case 2: $L_{u}=(3,1,1)$. Similarly, let $c_{1}, c_{2}$ and $c_{3}$ be the colors represented among the 5 colored neighbors of $u$, where $c_{1}$ appears three times. We now focus on vertices $v_{1}, w_{1}$ and $x_{1}$, the neighbors of $u$ having color $c_{1}$. Let us distinguish two cases.

(1) $v_{1}, w_{1}$ or $x_{1}$ is such that its colored neighbors use pairwise distinct colors. Then, similarly to Case 1 above, there are four other possibilities to color this vertex (no bicolored cycle can be created, and at most 4 colors should be avoided to maintain a proper coloring). Among those four colors, we pick a color $c$ such that $c \neq c_{2}$ and $c \neq c_{3}$, and we recolor the considered vertex with color $c$. In that case, $L_{u}$ is modified and becomes $L_{u}=(2,1,1,1)$, a case that is handled by Lemma 2 .

(2) $v_{1}, w_{1}$ and $x_{1}$ are such that at least one color is repeated among the colored neighbors of $v$. Then, by Observation 2, we conclude that $u$ should avoid (i) 4 colors (no bicolored cycles) and (ii) 3 more colors (proper coloring). Since we have 9 colors, there are always two colors available for $u$.

Case 3: $L_{u}=(4)$ (resp. $L_{u}=(4,1)$ ). Similarly, let $c_{1}$ be the color represented 4 times among the colored neighbors of $u$, and let us focus on vertices $v_{1}, w_{1}, x_{1}$ and $y_{1}$, the neighbors of $u$ having color $c_{1}$. Let us distinguish two cases.

(1) If $v_{1}, w_{1}, x_{1}$ or $y_{1}$ is such that its colored neighbors use pairwise distinct colors, then, as above, there are four other possibilities to color this vertex. Among those four colors, we pick a color $c$ such that is not represented among the neighbors of $u$, and we recolor the considered vertex with color $c$. In that case, $L_{u}$ is modified and becomes $L_{u}=(3,1)$ (resp. $\left.L_{u}=(3,1,1)\right)$, a case that is handled by Lemma 2 (resp. Case 2 above).

(2) If $v_{1}, w_{1}, x_{1}$ and $y_{1}$ are such that at least one color is repeated among the colored neighbors of $v$, then by Observation 2, we conclude that $u$ should avoid (i) 6 colors (no bicolored cycles) and (ii) at most 2 more colors (proper coloring). Since we have 9 colors, we conclude that there is always at least one color available for $u$.

Case 4: $L_{u}=(5)$. Similarly, let $c_{1}$ be the color assigned to the 5 colored neighbors of $u$. We now focus on vertices $v_{1}, w_{1}, x_{1}, y_{1}$ and $z_{1}$, the five neighbors of $u$, and we distinguish two cases.

(1) $v_{1}, w_{1}, x_{1}, y_{1}$ or $z_{1}$ is such that its colored neighbors use pairwise distinct colors. Then, as above, there are four other possibilities to color this vertex. Among those four colors, we pick a color $c$ such that $c \neq c_{1}$, and we and recolor the considered vertex with color $c$. In that case, $L_{u}$ is modified and becomes $L_{u}=(4,1)$, a case that is handled by Case 3 above.

(2) If $v_{1}, w_{1}, x_{1}, y_{1}$ and $z_{1}$ are such that at least one color is repeated among their colored neighbors, then by Observation 2, we conclude that $u$ should avoid (i) 7 colors (no bicolored cycles) and (ii) 1 more color (proper coloring). Since we have 9 colors, there is always one color available for $u$.

Lemmas 1, 2 and 3 cover almost all the possible cases for $u$. Only two cases cannot be solved by the methods describe above: $L_{u}=(2,2,1)$ and $L_{u}=(3,2)$. In order to be able to handle those two last cases, we first need the following technical lemma, which will prove useful later.

Lemma 4 Let $G$ be a partially colored graph of maximum degree 5, and let us suppose $G$ uses at most 9 colors. Then, for any colored vertex $x$ such that $\# c n(x) \leq 4$, there exists a way to recolor $x$ (with a different color) such that $G$ is still partially colored, and still uses at most 9 colors. 
Proof: Let $G$ be a partially colored graph of maximum degree 5, using at most 9 colors. Let $x$ be a colored vertex of $G$ such that $\# c n(x) \leq 4$.

Case 1: $\# \operatorname{cn}(x) \leq 3$. In that case, we know that we can always recolor $x$ in such a way that $G$ remains partially colored, and uses at most 9 colors. For this, see proof of Lemma 1, where $x$ plays the role of $u$, and where it was concluded that in each case, at least two choices exist for coloring $u$.

Case 2: $\# c n(x)=4$. We have 5 possibilities: $L_{x}=(1,1,1,1), L_{x}=(2,1,1), L_{x}=(3,1), L_{x}=(4)$ and $L_{x}=(2,2)$. Let us now detail those cases:

Case 2.1 $L_{x}=(1,1,1,1)$ or $L_{x}=(2,1,1)$. As above, proof of Lemma 2 yields that there are two choices to color $x$.

Case 2.2 $L_{x}=(3,1)$. Let $x_{1}, x_{2}$ and $x_{3}$ be the three neighbors of $x$ having the same color, say $c$, and let the second color be $c^{\prime}$. Let us look at any $x_{i}, 1 \leq i \leq 3: x_{i}$ has at most 5 colored neighbors, among which $x$. In the following, we will suppose that all 5 neighbors of $x_{i}$ are colored (if this is not the case, the result will also hold because there are less constraints on the problem).

(1) If, among the neighbors of $x_{i}$ (not considering vertex $x$ ), there is no color which is repeated, then $x_{i}$ sees $L_{x_{i}}=(1,1,1,1,1)$ or $L_{x_{i}}=(2,1,1,1)$ (depending on the color of $x$ ). What we want to show now is that in both cases, there are at least two choices to recolor $x_{i}$. If $L_{x_{i}}=(1,1,1,1,1)$, then $x_{i}$ just needs to avoid the colors of its 5 neighbors (there is no risk of a bicolored cycle involving $\left.x_{i}\right)$, and thus there are 4 choices for coloring $x_{i}$. If $L_{x_{i}}=(2,1,1,1)$, knowing that (i) $x$ (which is a neighbor of $x_{i}$ ) has only four colored neighbors, and (ii) there are two colored neighbors of $x$ which carry the same color $c$, we conclude by Observation 2 that $x_{i}$ should avoid at most (i) 2 colors (no bicolored cycles) and (ii) 4 more colors (proper coloring). Hence, in both cases, there are several choices to recolor $x_{i}$. Let us take any two of those choices, say $k_{1}$ and $k_{2}$. If $k_{1}=c^{\prime}$, then we recolor $x_{i}$ with $k_{2}$; else, we recolor $x_{i}$ with $k_{1}$. In both cases, $L_{x}$ is modified and becomes $L_{x}=(2,1,1)$, a case that has been treated in Case 2.1 above.

(2) In the case that all the $x_{i}$ s have at least one color which is repeated among their neighbors, then by Observation 2 we know that $x$ should avoid (i) at most 4 colors (no bicolored cycles) and (ii) 2 more colors (proper coloring). Thus, there are at least 3 choices to color $x$.

Case 2.3 $L_{x}=(4)$. Let $x_{1}, x_{2}, x_{3}$ and $x_{4}$ be the four colored neighbors of $x$, and let $c$ be their color. Let us focus on any $x_{i}, 1 \leq i \leq 4$, and as in the previous case, let us suppose that all 5 neighbors of $x_{i}$ are colored.

(1) If no color is repeated among the neighbors of $x_{i}$ (not taking $x$ into account), then $L_{x_{i}}=$ $(1,1,1,1,1)$ or $L_{x_{i}}=(2,1,1,1)$ (depending on the color of $\left.x\right)$. As in the previous case, we want to show that there is at least one possibility to recolor $x_{i}$ (there are actually more, but one will be enough for our purpose). Indeed, if $L_{x_{i}}=(1,1,1,1,1)$ then $x_{i}$ just needs to avoid the colors of its 5 neighbors (there is no risk of a bicolored cycle involving $x_{i}$ ), and thus there are 4 choices for coloring $x_{i}$. If $L_{x_{i}}=(2,1,1,1)$, knowing that (i) $x$ (which is a neighbor of $x_{i}$ ) has only four colored neighbors, and (ii) there are three colored neighbors of $x$ which carry the same color $c$, we conclude by Observation 2 that $x_{i}$ should avoid at most (i) 1 color (no bicolored cycles) and (ii) 4 more colors (proper coloring). Hence, in both cases, there are several choices to recolor $x_{i}$. Let us then recolor $x_{i}$ with any other available color for $x_{i}$. In that case, $L_{x}$ changes and becomes $L_{x}=(3,1)$, a case that has been treated in Case 2.2 above.

(2) In the case that all $x_{i}$ s have at least one repeated color among their neighbors, then by Observation 2 we know that $x$ should avoid (i) at most 6 colors (possible bicolored cycles) and (ii) 1 more color (proper coloring). Thus, there are at least 2 choices to color $x$, and thus at least one other choice than its current color.

Case 2.4 $L_{x}=(2,2)$. Let $x_{1}, x_{2}, x_{3}$ and $x_{4}$ be the four colored neighbors of $x$. Let us focus on a given $x_{i}$, for any $1 \leq i \leq 4$. As in the previous case, let us suppose that all 5 neighbors of $x_{i}$ are colored. 
(1) If no color is repeated among the neighbors of $x_{i}$ (not taking $x$ into account), then $L_{x_{i}}=$ $(1,1,1,1,1)$ or $L_{x_{i}}=(2,1,1,1)$ (depending on the color of $\left.x\right)$. As in the previous case, we want to show that there is at least one possibility to recolor $x_{i}$. Indeed, if $L_{x_{i}}=(1,1,1,1,1)$ then $x_{i}$ just needs to avoid the colors of its 5 neighbors (there is no risk of a bicolored cycle involving $x_{i}$ ), and thus there are 4 choices for coloring $x_{i}$. If $L_{x_{i}}=(2,1,1,1)$, knowing that (i) $x$ (which is a neighbor of $x_{i}$ ) has only four colored neighbors, and (ii) only two colors are used among the colored neighbors of $x$, we conclude by Observation 2 that $x_{i}$ should avoid at most (i) 2 colors (no bicolored cycles) and (ii) 4 more colors (proper coloring). Hence, in both cases, there are several choices to recolor $x_{i}$. Let us then recolor $x_{i}$ with any other available color for $x_{i}$. In that case, $L_{x}$ changes and becomes either $L_{x}=(3,1)$ or $L_{x}=(2,1,1)$, cases that have been treated in Case 2.1 and Case 2.2 above.

(2) In the case that all $x_{i}$ s have at least one repeated color among their neighbors, then by Observation 2 we know that $x$ should avoid (i) at most 6 colors (no bicolored cycles) and (ii) 2 more colors (proper coloring). Hence, in that case there is only one possibility to color $x$ and we cannot conclude using that argument. However, if we are in the situation where only one color is available for $x$, then for every $1 \leq i \leq 4$, the four neighbors of $x_{i}$ (not taking $x$ into account) take three different colors (ie, exactly one color is repeated, and repeated only once). Let $c_{i_{1}}, c_{i_{2}}$ and $c_{i_{3}}$ be those three colors, and let us again distinguish two cases: first, if $c(x)=c_{i_{1}}$ (resp. $c(x)=c_{i_{2}}, c(x)=c_{i_{3}}$ ), then $x$ can be recolored because color $c_{i_{1}}$ (resp. $c_{i_{2}}, c_{i_{3}}$ ) is taken into account into the 6 cycles we want to avoid ; but since $x$ has already been assigned this color, it should not be counted, which leaves room for another color for $x$.

Now suppose $c(x) \neq c_{i_{1}}, c_{i_{2}}, c_{i_{3}}$. Let us consider a vertex $x_{i}, 1 \leq i \leq 4$. We know that $L_{x_{i}}=(2,1,1,1)$, and let $w_{1}$ and $w_{2}$ be the two neighbors of $x_{i}$ having the same color, say c. Again, we look at the neighbors of $w_{j}(1 \leq j \leq 2)$. If $w_{j}$ has at least one repeated color among its neighbors (not taking $x_{i}$ into account), then $x_{i}$ can be recolored, because it should avoid (i) 3 colors (no bicolored cycles) and (ii) 4 more colors (proper coloring). Thus there is a second color available for $x_{i}$. Let us change the color of $x_{i}$. In that case, $L_{x}$ changes and becomes either $L_{x}=(3,1)$ or $L_{x}=(2,1,1)$, and we know that both cases can be solved (see Case 2.1 and Case 2.2 above).

Now if $w_{1}$ and $w_{2}$ have no repeated color among their neighbors, let us suppose (wlog) that all the neighbors of $w_{1}$ are colored, and that the colors of its neighbors are $\alpha_{1}, \alpha_{2}, \alpha_{3}, \alpha_{4}$. We will also show that it is possible to recolor $x_{i}$, and thus get to another case that has been previously solved. For this, we consider two more cases:

- $c\left(x_{i}\right)=\alpha_{j}$ for a $j \in[1 ; 4]$. As previously, $x_{i}$ has already been assigned a color that could create a bicolored cycle (though it does not create one, since we suppose $G$ is partially colored). Thus, when we count the number of colors $x_{i}$ should avoid, we should not take this possible bicolored cycle into account. Finally, we have that $x_{i}$ should avoid (i) 3 colors (no bicolored cycles) and (ii) 4 more colors (proper coloring), and thus $x_{i}$ can be recolored. In that case, $L_{x}$ changes and becomes either $L_{x}=(3,1)$ or $L_{x}=(2,1,1)$, and we know that both cases can be solved (see Case 2.1 and Case 2.2 above).

- $c\left(x_{i}\right) \neq \alpha_{j}$ for any $j \in[1 ; 4]$. In that case, $L_{w_{1}}=(1,1,1,1,1)$, and there are actually 4 choices to color $w_{1}$. If $c(x)$ is not among those choices, then there is a color $c^{\prime}$ that could be assigned to $w_{1}$ in such a way that $L_{x_{i}}=(1,1,1,1,1)$. In such a case, we recolor $w_{1}$ with $c^{\prime}$, and then we know that $x_{i}$ can be recolored as well. Now if $c(x)$ is among the choices of color for $w_{1}$, then we recolor $w_{1}$ with $c(x)$ and we now see that $x_{i}$ should avoid (i) 2 colors (no bicolored cycles, because of the neighbors of $x$ which only carry 2 different colors) and (ii) 4 more colors. Thus, there are several choices for coloring $x_{i}$, and consequently it can be recolored.

Altogether, we see that $x_{i}$ can be recolored, and thus $L_{x}$ either becomes $L_{x}=(3,1)$ or $L_{x}=(2,1,1)$, and we know that both cases can be solved (see Case 2.1 and Case 2.2 
above).

Lemma 5 Let $G$ be a partially colored graph of maximum degree 5, and let us suppose $G$ uses at most 9 colors. Then, for any uncolored vertex $u$, there exists a way to color $u$ such that $G$ is still partially colored, and still uses at most 9 colors.

Proof: Suppose $G$ is partially colored and uses at most 9 colors. Take any uncolored vertex $u$. If $\# c n(u) \leq 3$, then we know by Lemma 1 that we can assign a color to $u$ such that $G$ is still partially colored, and still uses at most 9 colors. Now suppose \#cn $(u)=4$. We have five cases for $L_{u}: L_{u}=(1,1,1,1), L_{u}=(2,1,1), L_{u}=(2,2), L_{u}=(3,1)$ and $L_{u}=(4)$. However, cases $L_{u}=(1,1,1,1), L_{u}=(2,1,1)$ and $L_{u}=(3,1)$ are covered by Lemma 2 , while $L_{u}=(2,2)$ and $L_{u}=(4)$ are covered by Lemma 3. Now suppose $\# \operatorname{cn}(u)=5$. We have seven cases for $L_{u}$ : $L_{u}=(1,1,1,1,1), L_{u}=(2,1,1,1), L_{u}=(2,2,1), L_{u}=(3,2), L_{u}=(3,1,1), L_{u}=(4,1)$ and $L_{u}=(5)$. However, cases $L_{u}=(1,1,1,1,1)$ and $L_{u}=(2,1,1,1)$ are covered by Lemma 2 , while cases $L_{u}=(3,1,1), L_{u}=(4,1)$ and $L_{u}=(5)$ are covered by Lemma 3 . Hence we are left with two cases: $L_{u}=(2,2,1)$ and $L_{u}=(3,2)$.

Case 1: $L_{u}=(2,2,1)$. Suppose the neighbors of $u$ are $v_{1}, w_{1}, v_{2}, w_{2}$ and $v_{3}$, such that $c\left(v_{1}\right)=$ $c\left(w_{1}\right)=c_{1}, c\left(v_{2}\right)=c\left(w_{2}\right)=c_{2}$ and $c\left(v_{3}\right)=c_{3}$. We recall that $c_{1}, c_{2}$ and $c_{3}$ are pairwise distinct colors. Thanks to Lemma 4 , we know that since $v_{1}$ is such that $\# c n\left(v_{1}\right) \leq 4$, it is possible to recolor it with a new color. Thus, let $c_{1}^{\prime} \neq c_{1}$ be the other color that is available for $v_{1}$. We have two cases:

(1) if $c_{1}^{\prime} \neq c_{3}$, then we recolor $v_{1}$, and $L_{u}$ becomes either $L_{u}=(3,1,1)$ (in case $c^{\prime}=c_{2}$ ) or $L_{u}=(2,1,1,1)$ (in case $\left.c^{\prime} \neq c_{2}\right)$. Each of those two cases are covered, respectively, by Lemma 3 and Lemma 2.

(2) if $c_{1}^{\prime}=c_{3}$, then even if we recolor $v_{1}, L_{u}$ will remain of the form $L_{u}=(2,2,1)$. In that case, we apply Lemma 4 on $v_{2}$ and we look at the other available color $c_{2}^{\prime} \neq c_{2}$ for $v_{2}$ :

- if $c_{2}^{\prime}=c_{1}$, then we recolor only $v_{2}$, which leads to $L_{u}=(3,1,1)$

- else, if $c_{2}^{\prime} \neq c_{3}$, then we recolor only $v_{2}$, which leads to $L_{u}=(2,1,1,1)$

- else, that is $c_{2}^{\prime}=c_{3}$, we recolor both $v_{1}$ and $v_{2}$, which leads to $L_{u}=(3,1,1)$

In each of the three above cases, Lemmas 3 and 2 show that we can color $u$ in such a way that at most 9 colors are used, and $G$ remains partially colored.

Case 2: Suppose now that $L_{u}=(3,2)$, and let us proceed similarly to Case 1. Indeed, suppose the neighbors of $u$ are $v_{1}, w_{1}, x_{1}, v_{2}$ and $w_{2}$, such that $c\left(v_{1}\right)=c\left(w_{1}\right)=c\left(x_{1}\right)=c_{1}$ and $c\left(v_{2}\right)=$ $c\left(w_{2}\right)=c_{2}$. We recall that $c_{1} \neq c_{2}$. Thanks to Lemma 4 , we know that since $v_{2}$ is such that $\# c n\left(v_{2}\right) \leq 4$, it is possible to recolor $v_{2}$ with a new color. Thus, let $c_{2}^{\prime} \neq c_{2}$ be the other color that is available for $v_{1}$. We have two cases: if $c_{2}^{\prime}=c_{1}$, then recoloring $v_{2}$ leads to the case $L_{u}=(4,1)$; else, recoloring $v_{2}$ leads to the case $L_{u}=(3,1,1)$. It can be seen that in both cases, Lemma 2 shows that we can color $u$ in such a way that at most 9 colors are used, and $G$ remains partially colored.

Thanks to the above results, we can state the following theorem.

Theorem 1 For any graph $G$ of maximum degree $5, a(G) \leq 9$ and there exists a linear-time algorithm that acyclically colors any such graph $G$ in at most 9 colors.

Proof: Lemmas 1, 2, 3 and 5 show that for any partially colored graph $G$ of maximum degree 5 that uses at most 9 colors, and for any uncolored vertex $u$, there exists a way to color $u$ in such a way that (i) $G$ remains partially colored and (ii) at most 9 colors are used.

The coloring algorithm that is implied by the above study is the following: starting from an 
uncolored graph $G$, arbitrarily pick an uncolored vertex $u$, and find a suitable color (among the 9 colors that are available) to color $u$, using the results of Lemmas 1, 2, 3 and 5 ; continue this process until all vertices are colored. Clearly, thanks to the above mentioned lemmas, we know that this algorithm is correct in the sense that it acyclically colors all the vertices of $G$, using at most 9 colors. Moreover, it runs in linear time since for each of the $n$ uncolored vertices considered, we need to look at distance at most 3 from it. Since we suppose that $\Delta=5$, in each of the $n$ loops, only a constant number of vertices is consulted. Consequently, the above greedy algorithm runs in $O(n)$.

\section{Conclusion}

In this paper, we have shown that any graph of maximum degree 5 can be acyclically colored with 9 colors, and we have provided a linear time algorithm that acyclically colors any such graph with at most 9 colors. This result can be seen as a follow-up of the ones obtained for graphs of maximum degree 3 (where we know $a(G) \leq 4$ for any such graph $G$ [Grü73]), and maximum degree 4 (where we know $a(G) \leq 5$ for any such graph $G$ [Bur79]).

As far as lower bounds are concerned, we know that there exists graphs $G$ of maximum degree 5 for which $a(G)=6$ (take for instance $K_{6}$ ). Closing the gap between those two bounds is a challenging open problem. In particular, we strongly suspect that the upper bound of 9 is not tight.

\section{References}

[AMR90] N. Alon, C. McDiarmid, and B. Reed, Acyclic colourings of graphs, Random Structures and Algorithms 2 (1990), 277-288.

[BKRS01] O.V. Borodin, A.V. Kostochka, A. Raspaud, and E. Sopena, Acyclic colouring of 1planar graphs, Discrete Applied Mathematics 114(1-3) (2001), 29-41.

[BKW99] O.V. Borodin, A.V. Kostochka, and D.R. Woodall, Acyclic colourings of planar graphs with large girth, J. London Math. Soc. 60(2) (1999), 344-352.

[Bor79] O.V. Borodin, On acyclic colorings of planar graphs, Discrete Mathematics 25 (1979), 211-236.

[Bur79] M.I. Burstein, Every 4-valent graph has an acyclic 5 coloring (in russian), Soobšč. Akad. Nauk Gruzin SSR 93 (1979), 21-24.

[FGR03] G. Fertin, E. Godard, and A. Raspaud, Acyclic and k-distance coloring of the grid, Information Processing Letters 87(1) (2003), 51-58.

[Grü73] B. Grünbaum, Acyclic colorings of planar graphs, Israel J. Math. 14(3) (1973), 390408.

[JMV06] R.E. Jamison, G.L. Matthews, and J. Villalpando, Acyclic colorings of products of trees, Information Processing Letters 99(1) (2006), 7-12.

[Sku04] S. Skulrattanakulchai, Acyclic colorings of subcubic graphs, Information Processing Letters 92(4) (2004), 161-167.

[Sop97] E. Sopena, The chromatic number of oriented graphs, Mathematical Notes 25 (1997), 191-205. 